\title{
Impulsive Synchronization of Nonlinear Stochastic Dynamical Networks with Time-Varying Delays
}

\author{
Li Li \\ Chengyi University College, Jimei University, Xiamen, China \\ Email: lilicy@jmu.edu.cn
}

How to cite this paper: Li, L. (2021) Impulsive Synchronization of Nonlinear Stochastic Dynamical Networks with Time-Varying Delays. Applied Mathematics, 12, 1145-1155. https://doi.org/10.4236/am.2021.1212073

Received: November 8, 2021

Accepted: December 6, 2021

Published: December 9, 2021

Copyright $\odot 2021$ by author(s) and Scientific Research Publishing Inc. This work is licensed under the Creative Commons Attribution International License (CC BY 4.0).

http://creativecommons.org/licenses/by/4.0/

\begin{abstract}
In this paper, an impulsive control strategy is proposed for a class of nonlinear stochastic dynamical networks with time-varying delay. Using the Lyapunov stability theory, a sufficient verifiable criterion for the exponential synchronization is derived analytically. Finally, a numerical simulation example is provided to verify the effectiveness of the proposed approach.
\end{abstract}

\section{Keywords}

Stochastic Dynamical Networks, Time-Varying Delays, Impulsive Control, Exponential Synchronization

\section{Introduction}

In recent years, complex networks have been widely studied due to their practical applications in different fields, such as the World Wide Web, genetic networks, biological neural networks, social networks, and so on [1]-[12].

Synchronization, as an interesting and important behavior of complex networks, has attracted much attention. In the past decades, some relevant theoretical results have been established [13]-[17]. As far as we all know, the dynamical network cannot realize synchronization by itself. For the purpose of driving the network to achieve synchronization, control inputs should be imposed on the nodes of dynamical networks. Until now, many useful control methods have been focused on this topic, such as adaptive control [18] [19], feedback control [20] [21], intermittent control [22], pinning control [23], and impulsive control [24] [25] [26]. In particularly the impulsive control has attracted more and more interests, which has been shown to be an effective control strategy in many fields due to its potential advantages over general continuous control schemes. Be- 
cause the impulsive control is more economical and can reduce the amount of the transmitted information.

Recently, synchronization of stochastic dynamical networks has aroused many interests [26] [27] [28] [29], since stochastic disturbances in complex networks need to be taken into account for modeling a realistic network. On the other hand, the time delay is ubiquitous in natural and man-made networks. The influence of a time delay increases the complexity of networks. [26] studied the synchronization of complex networks, which are discrete-time systems, with both time-varying delays and stochastic disturbances. [27] showed that impulsive controllers are effective for synchronization control of the stochastic dynamical networks, but time delay is not taken into account. In [28], the authors employed a pinning impulsive controller for the synchronization of time-delayed complex networks without random disturbance. To the best of our knowledge, very little work has been done on the impulsive synchronization of complex dynamical networks with both time-varying delays and stochastic disturbances.

The contributions of this paper are as follows. First, we study the nonlinear stochastic dynamical networks with time-varying delays, which are more general in the real world. Second, the exponential synchronization of such networks by impulsive control is investigated. A new sufficient verifiable criterion for the exponential synchronization is derived analytically and skillfully by applying the Lyapunov stability theory. A numerical simulation example is provided to verify the effectiveness of the proposed control method.

Notation: The standard notations will be used in this paper. $\mathbb{R}^{n}$ denotes the n-dimensional Euclidean space. $\mathbb{R}^{n} \times \mathbb{R}^{n}$ are $n \times n$ real matrices. $\|\cdot\|$ represents the Euclidean vector norm in $\mathbb{R}^{n}$. The superscript $\mathrm{T}$ represents the transpose. $\lambda_{\text {max }}(\cdot)$ represents the maximum eigenvalue of a matrix. For any random variable $\xi$, let $\mathbb{E}(\xi)$ be the expectation value of $\xi$.

\section{Prelimiaries}

In this paper, we consider the following dynamical network with time-varying delay.

$$
\begin{aligned}
\mathrm{d} x_{i}(t)= & {\left[\tilde{f}\left(t, x_{i}(t), x_{i}(t-\tau(t))\right)+c \sum_{j=1}^{N} a_{i j} \Gamma x_{j}(t)\right] \mathrm{d} t } \\
& +\tilde{\sigma}\left(t, x_{i}(t)\right) \mathrm{d} B(t), \quad i=1,2, \cdots, N
\end{aligned}
$$

where $x_{i}(t)=\left(x_{i 1}(t), x_{i 2}(t), \cdots, x_{i n}(t)\right)^{\mathrm{T}} \in \mathbb{R}^{n}$ is the state vector of $i$-th node at time $t, \quad(i=1,2, \cdots, N) . \tilde{f}\left(t, x_{i}(t), x_{i}(t-\tau(t))\right) \in \mathbb{R}^{n}$ is a nonlinear function describing the dynamics of nodes. $\tau(t)$ denotes the time-varying delay, which is bounded, continuous and differentiable, satisfying $0 \leq \tau(t) \leq \tau, \quad \dot{\tau}(t) \leq \tilde{\tau}<1$. Matrix $\Gamma=\operatorname{diag}\left(\gamma_{1}, \gamma_{2}, \cdots, \gamma_{n}\right)>0$ describes the inner coupling of the complex network. $c$ is a positive coupling strength of the network. The matrices

$A=\left(a_{i j}\right)_{N \times N}$ is the outer-coupling configuration of the network, in which $a_{i j}$ is defined as follows: if there is a connection between node $i$ and $j(j \neq i)$, then 
$a_{i j}=a_{j i}>0$, otherwise, $a_{i j}=a_{j i}=0$, and the diagonal elements are defined as $a_{i i}=-\sum_{j=1, j \neq i}^{N} a_{i j} . B(t) \in \mathbb{R}^{n}$ is an m-dimensional Brownian motion. $\tilde{\sigma}\left(t, x_{i}(t)\right): \mathbb{R} \times \mathbb{R}^{n} \rightarrow \mathbb{R}^{n \times m}$ is the noise intensity function matrix.

Assume $C\left([-\tau, 0], \mathbb{R}^{n}\right)$ be a Banach space of continuous functions mapping the interval $[-\tau, 0]$ into $\mathbb{R}^{n}$ with the norm $\|\phi\|=\sup _{-\tau \leq \theta \leq 0} \mathbb{E}(\|\phi(\theta)\|)$. The initial conditions associated with system (2.1) are given by $x_{i}(t)=\phi_{i}(t) \in C\left([-\tau, 0], \mathbb{R}^{n}\right)$.

Throughout this paper, we have the following assumptions.

Assumption 2.1. There exist two nonnegative constants $L_{1}$ and $L_{2}$, such that the nonlinear function $\tilde{f}(x, \bar{x})$ satisfies the following inequality

$$
(x-y)^{\mathrm{T}}(\tilde{f}(x, \bar{x})-\tilde{f}(y, \bar{y})) \leq L_{1}\|x-y\|^{2}+L_{2}\|\bar{x}-\bar{y}\|^{2}
$$

for any $x, y, \bar{x}, \bar{y} \in \mathbb{R}^{n}$.

Assumption 2.2. Assume that the noise intensity function matrix $\tilde{\sigma}\left(t, x_{i}(t)\right): \mathbb{R} \times \mathbb{R}^{n} \rightarrow \mathbb{R}^{n \times m}$ is uniformly Lipschitz continuous in terms of the norm induced by the inner product on the matrices

$$
\operatorname{trace}\left[(\tilde{\sigma}(t, u)-\tilde{\sigma}(t, v))^{\mathrm{T}} \cdot(\tilde{\sigma}(t, u)-\tilde{\sigma}(t, v))\right] \leq\|\mathfrak{M}(u-v)\|^{2}
$$

for any $u, v \in \mathbb{R}^{n}$, where $\mathfrak{M}$ is a known constant matrix with compatible dimensions.

Let $s(t)$ be a solution of an isolated node described by

$$
\mathrm{d} s(t)=\tilde{f}(t, s(t), s(t-\tau(t))) \mathrm{d} t+\tilde{\sigma}(t, s(t)) \mathrm{d} B(t) .
$$

In this paper, we want to control the nonlinear dynamical network (2.1) into the desired trajectory $s(t)$.

Let $e_{i}(t)=x_{i}(t)-s(t)$ be the error state of the node $i, i=1,2, \cdots, N$, then we have the following error dynamical system by subtracting Equation (2.2) from Equation (2.1):

$$
\begin{aligned}
\mathrm{d} e_{i}(t)= & {\left[\tilde{f}\left(t, x_{i}(t), x_{i}(t-\tau(t))\right)-\tilde{f}(t, s(t), s(t-\tau(t)))+c \sum_{j=1}^{N} a_{i j} \Gamma e_{j}(t)\right] \mathrm{d} t } \\
& +\sigma\left(t, e_{i}(t)\right) \mathrm{d} B(t), i=1,2, \cdots, N
\end{aligned}
$$

where $\sigma\left(t, e_{i}(t)\right)=\tilde{\sigma}\left(t, x_{i}(t)\right)-\tilde{\sigma}(t, s(t))$.

In order to force the whole network into the desired trajectory $s(t)$, the following impulsive controllers are designed.

$$
\left\{\begin{aligned}
\mathrm{d} e_{i}(t)= & {\left[\tilde{f}\left(t, x_{i}(t), x_{i}(t-\tau(t))\right)-\tilde{f}(t, s(t), s(t-\tau(t)))\right.} \\
& \left.+c \sum_{j=1}^{N} a_{i j} \Gamma e_{j}(t)\right] \mathrm{d} t+\sigma\left(t, e_{i}(t)\right) \mathrm{d} B(t), t \neq t_{k}, \\
e_{i}\left(t_{k}^{+}\right)= & e_{i}\left(t_{k}^{-}\right)+\mu e_{i}\left(t_{k}^{-}\right), t=t_{k} .
\end{aligned}\right.
$$

The discrete instant set $\left\{t_{k}\right\}$ denotes the impulsive sequences, which satisfies $t_{0}<t_{1}<\cdots<t_{k}<t_{k+1}<\cdots, \lim _{k \rightarrow+\infty} t_{k}=+\infty$.

The following definition is needed in this paper:

Definition 2.1. The solution of the dynamical system (2.4) is said to be expo- 
nential synchronization in mean square, if for an initial condition $e_{i}\left(t_{0}\right)(i=1,2, \cdots, N)$, there exist positive constants $\tilde{M}$ and $\varepsilon$ such that

$$
\mathbb{E}\left\{\sum_{i=1}^{N}\left\|e_{i}(t)\right\|^{2}\right\} \leq \tilde{M} \mathrm{e}^{-\varepsilon\left(t-t_{0}\right)}
$$

\section{Main Result}

In this section, we will derive the main results about our impulsive strategy for synchronization control of the nonlinear stochastic dynamical network (2.1) with time-varying delay. For convenience, we introduce the following notations. Let

$$
\omega=\frac{2 L_{2}}{1-\tilde{\tau}}, \rho=(1+\mu)^{2} .
$$

Theorem 3.1. Suppose that Assumption (2.1)-(2.2) hold, and $\tau \leq t_{k}-t_{k-1}$ for all $k=1,2, \cdots$, if there exists a positive constant $\varepsilon$ such that

$$
\rho+\omega \tau \leq \mathrm{e}^{-(\varepsilon+\hat{L})\left(t_{k}-t_{k-1}\right)},
$$

where

$$
\hat{L}=\omega+2 L_{1}+\lambda_{\max }\left(\mathfrak{M}^{\mathrm{T}} \mathfrak{M}\right),
$$

then the controlled dynamical network (2.4) is exponentially stable in mean square.

It means that the nonlinear stochastic dynamical network can be exponentially controlled to the objective trajectory $s(t)$ by using impulsive controllers (2.4).

Proof: According to the definition of exponential synchronization (2.1), and considering time-delays, we construct the following Lyapunov function:

$$
V(t)=V_{1}(t)+V_{2}(t) \text {, }
$$

where

$$
\begin{aligned}
& V_{1}(t)=\frac{1}{2} \sum_{i=1}^{N} e_{i}^{\mathrm{T}}(t) e_{i}(t), \\
& V_{2}(t)=\frac{1}{2} \omega \sum_{i=1}^{N} \int_{t-\tau(t)}^{t} e_{i}^{\mathrm{T}}(s) e_{i}(s) \mathrm{d} s .
\end{aligned}
$$

when $t \neq t_{k}$, the stochastic derivative of $V(t)$ can be obtained by Itô's differential formula [30].

$$
\begin{aligned}
& \mathrm{d} V(t)=\left\{\left[\frac{1}{2} \omega \sum_{i=1}^{N} e_{i}^{\mathrm{T}}(t) e_{i}(t)-\frac{\omega(1-\dot{\tau}(t))}{2} \sum_{i=1}^{N} e_{i}^{\mathrm{T}}(t-\tau(t)) e_{i}(t-\tau(t))\right]\right. \\
& +\sum_{i=1}^{N} e_{i}^{\mathrm{T}}(t)\left[\tilde{f}\left(t, x_{i}(t), x_{i}(t-\tau(t))\right)-\tilde{f}(t, s(t), s(t-\tau(t)))+c \sum_{j=1}^{N} a_{i j} \Gamma e_{j}(t)\right] \\
& \left.+\frac{1}{2} \sum_{i=1}^{N} \operatorname{trace}\left[\sigma^{\mathrm{T}}\left(t, e_{i}(t)\right) \sigma\left(t, e_{i}(t)\right)\right]\right\} \mathrm{d} t+\sum_{i=1}^{N} e_{i}^{\mathrm{T}}(t) \sigma\left(t, e_{i}(t)\right) \mathrm{d} B(t)
\end{aligned}
$$

By Assumptions (2.1) and (2.2), we have 


$$
\begin{aligned}
& \sum_{i=1}^{N} e_{i}^{\mathrm{T}}(t)\left[\tilde{f}\left(t, x_{i}(t), x_{i}(t-\tau(t))\right)-\tilde{f}(t, s(t), s(t-\tau(t)))\right] \\
& \leq L_{1} \sum_{i=1}^{N} e_{i}^{\mathrm{T}}(t) e_{i}(t)+L_{2} \sum_{i=1}^{N} e_{i}^{\mathrm{T}}(t-\tau(t)) e_{i}(t-\tau(t))
\end{aligned}
$$

and

$$
\frac{1}{2} \sum_{i=1}^{N} \operatorname{trace}\left[\sigma^{\mathrm{T}}\left(t, e_{i}(t)\right) \sigma\left(t, e_{i}(t)\right)\right] \leq \frac{1}{2} \lambda_{\max }\left(\mathfrak{M}^{\mathrm{T}} \mathfrak{M}\right) \sum_{i=1}^{N} e_{i}^{\mathrm{T}}(t) e_{i}(t) .
$$

It follows from the diffusive property of symmetric matrix $A$ that

$$
\begin{aligned}
c \sum_{i=1}^{N} \sum_{j=1}^{N} a_{i j} e_{i}^{\mathrm{T}}(t) \Gamma e_{j}(t) & =c \sum_{i=1}^{N} \sum_{j=1}^{N} a_{i j}\left[\sum_{\theta=1}^{n} e_{i \theta}(t) \gamma_{\theta} e_{j \theta}(t)\right] \\
& =c \sum_{\theta=1}^{n} \gamma_{\theta}\left[\sum_{i=1}^{N} \sum_{j=1}^{N} a_{i j} e_{i \theta}(t) e_{j \theta}(t)\right] \\
& =-\frac{1}{2} c \sum_{\theta=1}^{n} \sum_{i=1}^{N} \sum_{j=1, j \neq i}^{N} \gamma_{\theta} a_{i j}\left(e_{i \theta}(t)-e_{j \theta}(t)\right)^{2} \\
& \leq 0 .
\end{aligned}
$$

Since $\dot{\tau}(t) \leq \tilde{\tau}<1$, considering (3.4)-(3.6), the following inequality can be obtained:

$$
\begin{aligned}
\mathrm{d} V(t) \leq & \left\{\left[\frac{1}{2} \omega \sum_{i=1}^{N} e_{i}^{\mathrm{T}}(t) e_{i}(t)-\frac{1}{2} \omega(1-\tilde{\tau}) \sum_{i=1}^{N} e_{i}^{\mathrm{T}}(t-\tau(t)) e_{i}(t-\tau(t))\right]\right. \\
& +\sum_{i=1}^{N} L_{1} e_{i}^{\mathrm{T}}(t) e_{i}(t)+\sum_{i=1}^{N} L_{2}\left(e_{i}^{\mathrm{T}}(t-\tau(t)) e_{i}(t-\tau(t))\right) \\
& \left.+\frac{1}{2} \lambda_{\max }\left(\mathfrak{M}^{\mathrm{T}} \mathfrak{M}\right) \sum_{i=1}^{N} e_{i}^{\mathrm{T}}(t) e_{i}(t)\right\} \mathrm{d} t+\sum_{i=1}^{N} e_{i}^{\mathrm{T}}(t) \sigma\left(t, e_{i}(t)\right) \mathrm{d} B(t) .
\end{aligned}
$$

Taking the mathematical expectation, we obtained

$$
\mathbb{E}\left\{\frac{\mathrm{d} V(t)}{\mathrm{d} t}\right\} \leq \mathbb{E}\left\{\hat{L} V_{1}(t)\right\} \leq \mathbb{E}\{\hat{L} V(t)\},
$$

where $\hat{L}=\omega+2 L_{1}+\lambda_{\max }\left(\mathfrak{M}^{\mathrm{T}} \mathfrak{M}\right)$.

From (3.8), for $t \in\left(t_{k-1}, t_{k}\right)$, we have

$$
\mathbb{E}\{V(t)\} \leq \mathbb{E}\left\{V\left(t_{k-1}^{+}\right)\right\} \mathrm{e}^{\hat{L}\left(t-t_{k-1}\right)} .
$$

For $t=t_{k}$, we have

$$
\begin{aligned}
& \mathbb{E}\left\{V_{1}\left(t_{k}^{+}\right)\right\}=\mathbb{E}\left\{\frac{1}{2} \sum_{i=1}^{N}(1+\mu)^{2} e_{i}^{\mathrm{T}}\left(t_{k}^{-}\right) e_{i}\left(t_{k}^{-}\right)\right\} \triangleq \rho \mathbb{E}\left\{V_{1}\left(t_{k}^{-}\right)\right\}, \\
& \mathbb{E}\left\{V_{2}\left(t_{k}^{+}\right)\right\}=\mathbb{E}\left\{\frac{1}{2} \omega \sum_{i=1}^{N} \int_{t_{k}^{+}-\tau\left(t_{k}^{+}\right)}^{t_{k}^{+}} e_{i}^{\mathrm{T}}(s) e_{i}(s) \mathrm{d} s\right\}=\mathbb{E}\left\{V_{2}\left(t_{k}^{-}\right)\right\} .
\end{aligned}
$$

Next, we will use mathematical induction to show that the following inequality holds.

$$
\mathbb{E}\{V(t)\} \leq M \mathrm{e}^{-(\varepsilon+\hat{L})\left(t_{k}-t_{0}\right)} \mathrm{e}^{\hat{L}\left(t-t_{0}\right)}, t \in\left(t_{k-1}, t_{k}\right] \text {, for } k=1,2, \cdots
$$

where $M=\mathbb{E}\left\{V\left(t_{0}^{+}\right)\right\} \mathrm{e}^{(\varepsilon+\hat{L})\left(t_{1}-t_{0}\right)}$. 
First, for $t \in\left(t_{0}, t_{1}\right]$, according to (3.9), we can get

$$
\begin{aligned}
\mathbb{E}\{V(t)\} & \leq \mathbb{E}\left\{V\left(t_{0}^{+}\right)\right\} \mathrm{e}^{\hat{L}\left(t-t_{0}\right)} \\
& =\mathbb{E}\left\{V\left(t_{0}^{+}\right)\right\} \mathrm{e}^{(\varepsilon+\hat{L})\left(t_{1}-t_{0}\right)} \mathrm{e}^{-(\varepsilon+\hat{L})\left(t_{1}-t_{0}\right)} \mathrm{e}^{\hat{L}\left(t-t_{0}\right)} \\
& \triangleq M \mathrm{e}^{-(\varepsilon+\hat{L})\left(t_{1}-t_{0}\right)} \mathrm{e}^{\hat{L}\left(t-t_{0}\right)}
\end{aligned}
$$

Then, (3.10) and (3.13) imply that

$$
\mathbb{E}\left\{V_{1}\left(t_{1}^{+}\right)\right\}=\rho \mathbb{E}\left\{V_{1}\left(t_{1}^{-}\right)\right\} \leq \rho \mathbb{E}\left\{V\left(t_{1}^{-}\right)\right\} \leq \rho M \mathrm{e}^{-(\varepsilon+\hat{L})\left(t_{1}-t_{0}\right)} \mathrm{e}^{\hat{L}\left(t_{1}-t_{0}\right)}
$$

Since $\tau \leq t_{1}-t_{0}$, it follows from (3.11) and (3.14) that

$$
\begin{aligned}
\mathbb{E}\left\{V_{2}\left(t_{1}^{+}\right)\right\} & =\mathbb{E}\left\{V_{2}\left(t_{1}\right)\right\}=\mathbb{E}\left\{\frac{1}{2} \omega \sum_{i=1}^{N} \int_{t_{1}-\tau\left(t_{1}\right)}^{t_{1}} e_{i}^{\mathrm{T}}(s) e_{i}(s) \mathrm{d} s\right\} \\
& \leq \mathbb{E}\left\{\frac{1}{2} \omega \sum_{i=1}^{N} \int_{t_{1}-\tau}^{t_{1}} e_{i}^{\mathrm{T}}(s) e_{i}(s) \mathrm{d} s\right\} \\
& \leq \frac{1}{2} \omega \tau \mathbb{E}\left\{\sup _{s \in\left[t_{1}-\tau, t_{1}\right]}\left\{\sum_{i=1}^{N} e_{i}^{\mathrm{T}}(s) e_{i}(s)\right\}\right\} \\
& =\omega \tau \mathbb{E}\left\{\sup _{s \in\left[t_{1}-\tau, t_{1}\right]}\left\{V_{1}(s)\right\}\right\} \\
& \leq \omega \tau M \mathrm{e}^{-(\varepsilon+\hat{L})\left(t_{1}-t_{0}\right)} \mathrm{e}^{\hat{L}\left(t_{1}-t_{0}\right)}
\end{aligned}
$$

From condition (3.1)

$$
\begin{aligned}
\mathbb{E}\left\{V\left(t_{1}^{+}\right)\right\} & =\mathbb{E}\left\{V_{1}\left(t_{1}^{+}\right)+V_{2}\left(t_{1}^{+}\right)\right\} \\
& \leq(\rho+\omega \tau) M \mathrm{e}^{-(\varepsilon+\hat{L})\left(t_{1}-t_{0}\right)} \mathrm{e}^{\hat{L}\left(t_{1}-t_{0}\right)} \\
& \leq M \mathrm{e}^{-(\varepsilon+\hat{L})\left(t_{2}-t_{0}\right)} \mathrm{e}^{\hat{L}\left(t_{1}-t_{0}\right)} .
\end{aligned}
$$

Then, for $t \in\left(t_{1}, t_{2}\right]$, we have

$$
\mathbb{E}\{V(t)\} \leq \mathbb{E}\left\{V\left(t_{1}^{+}\right)\right\} \mathrm{e}^{\hat{L}\left(t-t_{1}\right)} \leq M \mathrm{e}^{-(\varepsilon+\hat{L})\left(t_{2}-t_{0}\right)} \mathrm{e}^{\hat{L}\left(t-t_{0}\right)} .
$$

Assume that for $t \in\left(t_{n-1}, t_{n}\right],(n>2)$, the following inequality holds:

$$
\mathbb{E}\{V(t)\} \leq M \mathrm{e}^{-(\varepsilon+\hat{L})\left(t_{n}-t_{0}\right)} \mathrm{e}^{\hat{L}\left(t-t_{0}\right)} .
$$

Similar to the discussion in (3.14)-(3.16), we can get

$$
\begin{aligned}
\mathbb{E}\left\{V\left(t_{n}^{+}\right)\right\} & =\mathbb{E}\left\{V_{1}\left(t_{n}^{+}\right)+V_{2}\left(t_{n}^{+}\right)\right\} \\
& \leq(\rho+\omega \tau) M \mathrm{e}^{-(\varepsilon+\hat{L})\left(t_{n}-t_{0}\right)} \mathrm{e}^{\hat{L}\left(t_{n}-t_{0}\right)} \\
& \leq M \mathrm{e}^{-(\varepsilon+\hat{L})\left(t_{n+1}-t_{0}\right)} \mathrm{e}^{\hat{L}\left(t_{n}-t_{0}\right)} .
\end{aligned}
$$

Then, for $t \in\left(t_{n}, t_{n+1}\right]$,

$$
\mathbb{E}\{V(t)\} \leq \mathbb{E}\left\{V\left(t_{n}^{+}\right)\right\} \mathrm{e}^{\hat{L}\left(t-t_{n}\right)} \leq M \mathrm{e}^{-(\varepsilon+\hat{L})\left(t_{n+1}-t_{0}\right)} \mathrm{e}^{\hat{L}\left(t-t_{0}\right)} .
$$

Thus, the inequality (3.12) has been proved.

Base on inequality (3.12), for $t \in\left(t_{k-1}, t_{k}\right]$, it is easy to check that

$$
\begin{aligned}
\mathbb{E}\{V(t)\} & \leq M \mathrm{e}^{-(\varepsilon+\hat{L})\left(t_{k}-t_{0}\right)} \mathrm{e}^{\hat{L}\left(t-t_{0}\right)} \leq M \mathrm{e}^{-(\varepsilon+\hat{L})\left(t_{k}-t_{0}\right)} \mathrm{e}^{\hat{L}\left(t_{k}-t_{0}\right)} \\
& =M \mathrm{e}^{-\varepsilon\left(t_{k}-t_{0}\right)} \leq M \mathrm{e}^{-\varepsilon\left(t-t_{0}\right)} .
\end{aligned}
$$


Thus, from the construction of $V(t)$, we have

$$
\mathbb{E}\left\{\sum_{i=1}^{N}\left\|e_{i}(t)\right\|^{2}\right\} \leq \tilde{M} \mathrm{e}^{-\varepsilon\left(t-t_{0}\right)},
$$

where $\tilde{M}=2 M$. Then the controlled dynamical network (2.4) is said to be exponentially stable in mean square. In addition, it can be seen from the proof process that the initial conditions do not affect the final conclusion.

\section{Numerical Example}

In this section, a numerical example will be given to demonstrate the effectiveness of our main results. A time-delayed Lorenz system with Brownian noise is selected as the isolated node of the dynamical network, and the dynamic network system we considered has 5 nodes. The $i$-th node $(i=1,2, \cdots, 5)$ is described as follows:

$\left\{\begin{array}{l}\mathrm{d} x_{i 1}(t)=\left[a\left(x_{i 2}(t)-x_{i 1}(t)\right)+d\left(x_{i 1}(t-\tau(t))-x_{i 1}(t)\right)\right] \mathrm{d} t+0.2 \cdot\left\|x_{i}(t)\right\| \cdot \mathrm{d} B_{1}(t), \\ \mathrm{d} x_{i 2}(t)=\left[b x_{i 1}(t)-x_{i 1}(t) x_{i 3}(t)-x_{i 2}(t)\right] \mathrm{d} t+0.2 \cdot\left\|x_{i}(t)\right\| \cdot \mathrm{d} B_{2}(t), \\ \mathrm{d} x_{i 3}(t)=\left[x_{i 1}(t) x_{i 2}(t)-c x_{i 3}(t)\right] \mathrm{d} t+0.2 \cdot\left\|x_{i}(t)\right\| \cdot \mathrm{d} B_{3}(t),\end{array}\right.$

where $\mathrm{d} B_{i}(t)$ is a 3-D Brownian motion, $a=10, b=28, c=8 / 3, d=5$ and the time-varying delay $\tau(t)=\frac{0.01 \mathrm{e}^{t}}{1+\mathrm{e}^{t}}$. Note that $\tau(t) \leq 0.01$ and $\dot{\tau}(t) \leq 0.0025$, thus we have $\tau=0.01$ and $\tilde{\tau}=0.0025$. Then we get $L_{1}=80.998$ and $L_{2}=0.5$ for Assumption 2.1. Provided no Brownian motion noise, systems (4.1) has a chaotic attractor with initial value $[-10,2,-3]$ as shown in Figure 1.

The system parameters are chosen as follows: the coupling strength $c=0.35$, the inner coupling matrix $\Gamma$ as the identity matrix and the outer coupling matrice

$$
\left(a_{i j}\right)_{5 \times 5}=\left(\begin{array}{ccccc}
-3 & 1 & 1 & 0 & 1 \\
1 & -4 & 1 & 1 & 1 \\
1 & 1 & -4 & 1 & 1 \\
0 & 1 & 1 & -3 & 1 \\
0 & 1 & 1 & 1 & -3
\end{array}\right)
$$

The impulsive controller (2.4) is adopted here with $t_{k}-t_{k-1}=0.002$ and $\mu=-0.2$. By simple calculations, we get that $\omega=1.0025, \hat{L}=164.13$ and $\varepsilon=43.62$. Figure 2 displays the orbits of norms of $\left\|e_{i}(t)\right\|^{2}(i=1,2, \cdots, 5)$ with the initial conditions

$$
x_{i}(t)=\left(\begin{array}{c}
-8+4 i \cos t \\
12+5 i \cos t \\
2-2 i \cos t
\end{array}\right), t \in[-0.5,0]
$$

It shows that the time-varying delayed network considered here achieved exponential synchronization by the proposed impulsive strategy. 


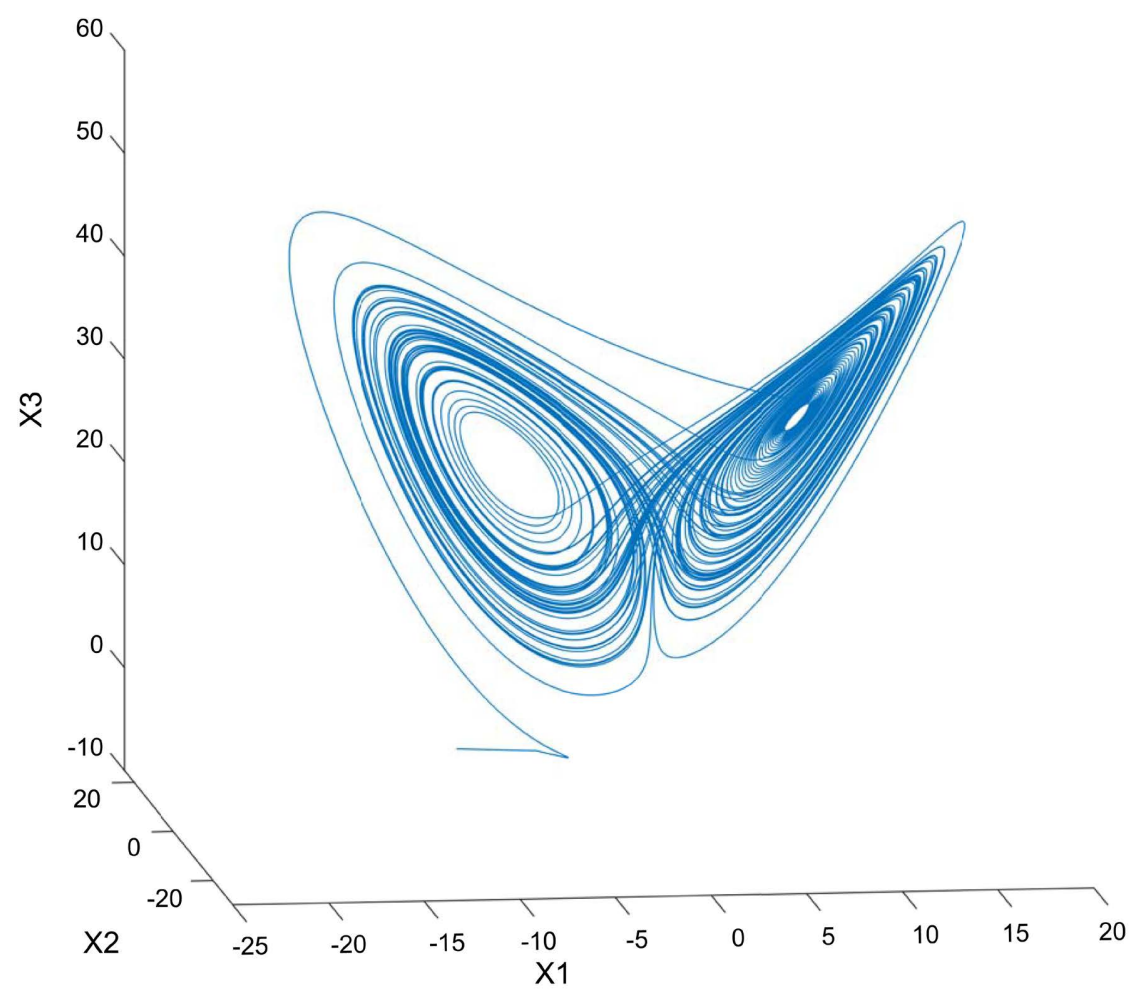

Figure 1. Phase trajectories of Lorenz system without noise.

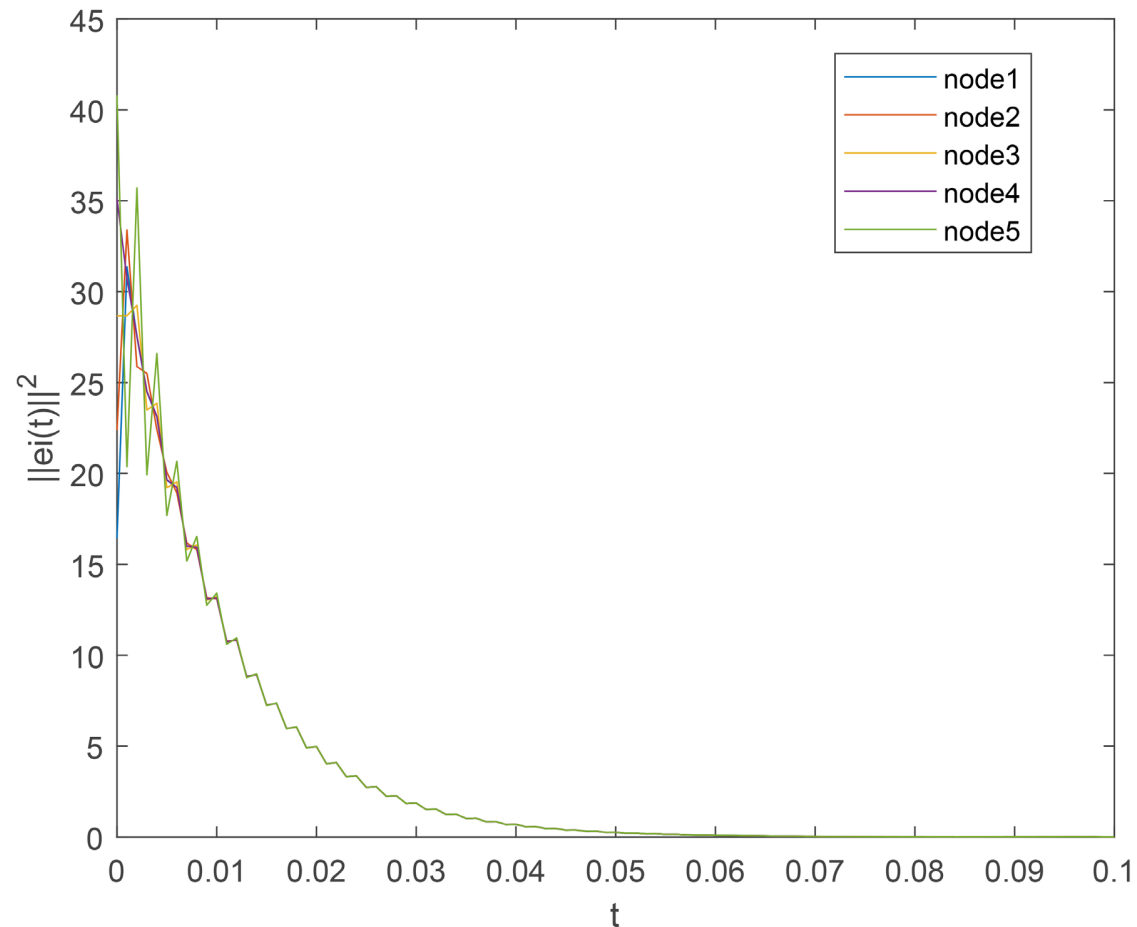

Figure 2. Trajectories of the synchronization errors $\left(\left\|e_{i}(t)\right\|^{2}\right)$.

\section{Conclusion}

An impulsive control strategy is proposed for the synchronization of nonlinear 
stochastic dynamical networks with time-varying delay in this paper. By applying the Lyapunov stability theory and mathematical analysis technique, sufficient verifiable criterion for the exponential synchronization is derived analytically. It is shown that synchronization can be achieved by impulsively controlling the network nodes. Finally, a numerical simulation is used to demonstrate the effectiveness of our method. For the future work, the author will study the finite-time synchronization of stochastic complex networks with time delays and uncertain disturbance.

\section{Funding}

This work was supported by the education and scientific research project for middle-aged and young teachers of Fujian Province (No. JAT191151).

\section{Conflicts of Interest}

The author declares no conflicts of interest regarding the publication of this paper.

\section{References}

[1] Ott, E., Grebogi, C. and Yorke, J.A. (1993) Controlling Chaos. Physical Review Letters, 64, 1196-1199. https://doi.org/10.1103/PhysRevLett.64.1196

[2] Newman, M. and Walls, D.J. (1999) Scaling and Percolation in the Small-World Network Model. Physical Review E, 60, 7332-7342. https://doi.org/10.1103/PhysRevE.60.7332

[3] Wang, Y., Wang, Z. and Liang, J. (2008) A Delay Fractioning Approach to Global Synchronization of Delayed Complex Networks with Stochastic Disturbances. Physical Letters A, 372, 6066-6073. https://doi.org/10.1016/j.physleta.2008.08.008

[4] Yang, J., Lu, L., Xie, W., Chen, G. and Dong, Z. (2007) On Competitive Relationship Networks: A New Method for Industrial Competition Analysis. Physica A: Statistical Mechanics and Its Applications, 382, 704-714. https://doi.org/10.1016/j.physa.2007.04.032

[5] Hu, A. and Xu, Z. (2009) Pinning a Complex Dynamical Network via Impulsive Control. Physics Letters A, 374, 186-190. https://doi.org/10.1016/j.physleta.2009.10.049

[6] Yang, J., Yao, C., Ma, W. and Chen, G. (2010) A Study of the Spreading Scheme for Viral Marketing Based on a Complex Network Model. Physica A: Statistical Mechanics and Its Applications, 389, 859-870. https://doi.org/10.1016/j.physa.2009.10.034

[7] Watts, D.J. and Strogatz, S.H. (1998) Collective Dynamic of Small World Network. Nature, 393, 440-442. https://doi.org/10.1038/30918

[8] Barabasi, A.L. and Albert, R. (1999) Emergence of Scaling in Random Networks. Science, 286, 509-512. https://doi.org/10.1126/science.286.5439.509

[9] Newman, M. (2003) The Structure and Function of Complex Networks. Siam Review, 45, 167-256. https://doi.org/10.1137/S003614450342480

[10] Wang, X. and Chen, G. (2003) Complex Networks: Small-World, Scale-Free and beyond. IEEE Circuits and Systems Magazine, 3, 6-20.

https://doi.org/10.1109/MCAS.2003.1228503 
[11] Wang, X., Li, X. and Chen, G. (2006) Theory and Application of Complex Networks. Tsinghua University Press, Beijing.

[12] Zhu, H., Luo, H., Peng, H., Li, L. and Luo, Q. (2009) Complex Networks-Based Energy-Efficient Evolution Model for Wireless Sensor Networks. Chaos, Solitons and Fractals, 4, 1828-1835. https://doi.org/10.1016/j.chaos.2008.07.032

[13] Arenas, A., Daz-Guilera, A., et al. (2008) Synchronization in Complex Networks. Physics Reports, 469, 93-153. https://doi.org/10.1016/j.physrep.2008.09.002

[14] Anbuvithya, R., Mathiyalagan, K., Sakthivel, R. and Prakash, P. (2015) Non-Fragile Synchronization of Memristive BAM Networks with Random Feedback Gain Fluctuations. Communications in Nonlinear Science and Numerical Simulation, 29, 427-440. https://doi.org/10.1016/j.cnsns.2015.05.020

[15] Li, H.L., Hu, C., Cao, J., Jiang, H. and Alsaedi, A. (2019) Quasi-Projective and Complete Synchronization of Fractional-Order Complex-Valued Neural Networks with Time Delays. Neural Networks, 118, 102-109. https://doi.org/10.1016/j.neunet.2019.06.008

[16] Qiu, X., Lin, W. and Zheng, Y. (2020) Function Projective Synchronization of Complex Networks with Distributed Delays via Hybrid Feedback Control. IEEE Access, 8, 99110-99114. https://doi.org/10.1109/ACCESS.2020.2998359

[17] Ma, Y. and Tai, Y. (2020) Finite-Time Synchronization for Heterogeneous Complex Networks with Time-Varying Delays. Applied Mathematics, 11, 1000-1012. https://doi.org/10.4236/am.2020.1110066

[18] Sastry, S. and Bodson, M. (1990) Adaptive Control: Stability, Convergence and Robustness. Journal of the Acoustical Society of America, 88, 588-589. https://doi.org/10.1121/1.399905

[19] Liu, X. (2020) Distributed Synchronization of Coupled Time-Delay Neural Networks Based on Randomly Occurring Control. Applied Mathematics, 11, 698-711. https://doi.org/10.4236/am.2020.117047

[20] Corriou, J.P. (2004) Process Control. Springer, London. https://doi.org/10.1007/978-1-4471-3848-8

[21] Yassen, M.T. (2005) Controlling Chaos and synchronization for New Chaotic System Using Linear Feedback Control. Chaos, Solitons and Fractals, 26, 913-920. https://doi.org/10.1016/j.chaos.2005.01.047

[22] Jing, T., Zhang, D., Mei, J. and Fan, Y. (2019) Inite-Time Synchronization of Delayed Complex Dynamic Networks via Aperiodically Intermittent Control. Journal of the Franklin Institute, 356, 5464-5484. https://doi.org/10.1016/j.jfranklin.2019.03.024

[23] Wang, X. and Chen, G. (2002) Pinning Control of Scale-Free Dynamical Networks, Physica A: Statistical Mechanics and Its Applications, 310, 521-531. https://doi.org/10.1016/S0378-4371(02)00772-0

[24] Tao, Y. (1999) Impulsive Control. IEEE Transactions on Automatic Control, 44, 1081-1083. https://doi.org/10.1109/9.763234

[25] Zhou, J., Xiang, L. and Liu, Z. (2007) Synchronization in complex Delayed Dynamical Networks with Impulsive Effects. Physica A: Statistical Mechanics and Its Applications, 384, 684-692. https://doi.org/10.1016/j.physa.2007.05.060

[26] Zhang, Q., Chen, G. and Wan, L. (2018) Exponential Synchronization of Discrete-Time Impulsive Dynamical Networks with Time-Varying Delays and Stochastic Disturbances. Neurocomputing, 309, 62-69. https://doi.org/10.1016/j.neucom.2018.04.070 
[27] Lu, J., Kurths, J., Cao, J., Mahdavi, N. and Chi, H. (2012) Synchronization Control for Nonlinear Stochastic Dynamical Networks: Pinning Impulsive Strategy. IEEE Transactions on Neural Networks and Learning Systems, 23, 285-292. https://doi.org/10.1109/TNNLS.2011.2179312

[28] Xin, W., Liu, X., She, K. and Zhong, S. (2017) Pinning Impulsive Synchronization of Complex Dynamical Networks with Various Time-Varying Delay Sizes. Nonlinear Analysis. Hybrid Systems, 26, 307-318. https://doi.org/10.1016/j.nahs.2017.06.005

[29] Tang, Y., Wong, W., Fang, J. and Miao, Q. (2011) Pinning Impulsive Synchronization of Stochastic Delayed Coupled Networks. Chinese Physics B, 20, Article ID: 040513. https://doi.org/10.1088/1674-1056/20/4/040513

[30] Mao, X. (2007) Stochastic Differential Equations and Applications. Horwood Publishing, Chichester. https://doi.org/10.1533/9780857099402 